\title{
CORRELAÇÃO ENTRE A DINAMOMETRIA E TESTE FUNCIONAL EM ATLETAS DE HANDEBOL
}

CORRELATION BETWEEN DYNAMOMETRYAND FUNCTIONAL TEST IN HANDBALL ATHLETES

CORRELACIÓN ENTRE DINAMOMETRÍA Y PRUEBA FUNCIONAL EN ATLETAS DE BALONMANO

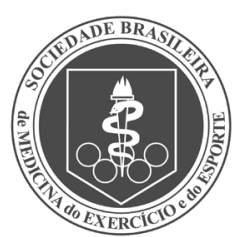

Artigo Original

\author{
Fabiano Fernandes da Silva $a^{1,2}$ \\ (Educador Físico) \\ Ciro Augusto Fernandes de \\ Oliveira Penido' (Farmacêutico) \\ Valter Luis Pereira Junior' (Médico) \\ Renato Aparecido de Souza ${ }^{1,2}$ \\ (Fisioterapeuta) \\ Antonio Balbin Villaverde' (Físico) \\ 1. Instituto de Engenharia \\ Biomédica da Universidade \\ Camilo Castelo Branco, \\ UNICASTELO, São José dos \\ Campos, SP, Brasil. \\ 2. Grupo de Estudos e Pesquisa \\ em Ciências da Saúde. Instituto \\ Federal de Educação Ciência \\ e Tecnologia do Sul de Minas \\ Gerais, Campus Muzambinho, \\ MG, Brasil.
}

\section{Correspondência:}

Rua Dinah, 75, Bairro Canaã. 37890-000 Muzambinho, MG, Brasil.professor.fabiano@yahoo. com.br

\section{RESUMO}

Introdução: São poucas as informações que relacionam testes funcionais com testes mais precisos, como a dinamometria isocinética. Objetivo: Correlacionar as variáveis isocinéticas pico de torque (PT), potência média (PM) e trabalho total (TT) de rotadores internos de ombro em atletas de handebol do gênero masculino com o teste funcional de arremesso de medicine ball (AMB). Métodos: Participaram desse estudo 25 atletas amadores de handebol do gênero masculino com idade média 17,72 $\pm 2,16$ anos. A coleta de dados foi realizada em dois dias diferentes, respeitando intervalo mínimo de 48 horas entre as sessões. De maneira aleatória, definida por sorteio, os atletas faziam o teste isocinético ou o teste de AMB. Para o teste de AMB, o atleta deveria arremessar uma medicine ball de $3 \mathrm{~kg}$ a maior distância possível. Para análise isocinética, foram utilizadas as velocidades de $60 \% \mathrm{se}$ $180 \%$ avaliando a capacidade muscular dos músculos rotadores internos de ombro. Para análise de associações foram utilizados os testes de correlação de Pearson e regressão linear simples. Resultados: Foram observadas associações moderadas $(0,60 \leq r<0,70 ; p<0,05)$ para as variáveis PT, PM e TT e o teste de AMB na velocidade de $60 \%$ s. Além disso, observaram-se associações altas $(r \geq 0,70 ; p<0,05)$ entre as variáveis estudadas na velocidade de 180\% s. Conclusão: As variáveis isocinéticas PT, PM e TT, de rotadores internos de ombro do braço dominante de atletas de handebol do gênero masculino apresentam correlação moderada a alta com o teste funcional de AMB.

Palavras-chave: ombro, torque, dinamômetro de força muscular.

\section{ABSTRACT}

Introduction: There is little information that correlates the functional tests with more accurate tests such as isokinetic dynamometry. Objective: To correlate the isokinetic variables peak torque (PT), mean power (MP) and total work (TW) of the internal rotators of the shoulder in male handball athletes, through the medicine ball throw (MBT) functional test. Methods: 25 male, amateur handball athletes, with an average age of $17.72 \pm 2.16$ years, took part in this study. The data collection was performed on two different days, with an interval of 48 hours between sessions. The athletes were assigned by a random draw, to perform the isokinetic assessment or the MBT test. For the MBT test, the athlete was instructed to throw a medicine ball (3kg) as far as possible. For the isokinetic analysis, speeds of $60 \% \mathrm{~s}$ and $180 \%$ s were used, evaluating the muscular capacity of the internal rotator muscles of the shoulder. To analyze the associations, Pearson's correlation and simple linear regression were used. Results: Moderate associations $(0.60 \leq r<0.70 ; p<0.05)$ were observed for the variables PT, MP and TW with the MBT test at the speed of $60 \%$. Also, high correlations $(r \geq 0.70 ; p<0.05)$ were observed between the variables studied at the speed of $180 \%$. Conclusion: The isokinetic variables PT, MP and TW of the internal rotators of the shoulder of the dominant arm in male handball athletes show a moderate and high correlation with the functional MBT.

Keywords: shoulder, torque, muscle strength dynamometer.

\section{RESUMEN}

Introducción: Existe poca información que correlacionen pruebas funcionales con pruebas más precisas, como el dinamómetro isocinético. Objetivo: Correlacionar las variables de torque máximo (PT), la potencia media (PM) y el trabajo total (TT) de los rotadores internos del hombro con la prueba funcional de lanzamiento de medicine ball (AMB) en atletas de balonmano del sexo masculino. Métodos: Participaron del estudio 25 atletas de balonmano masculino con una edad media 17,72 2, 216 años. La recolección de datos se llevó a cabo en dos días diferentes, respetando un intervalo mínimo de 48 horas entre las sesiones. Los atletas realizaron test isocinético o prueba de AMB de manera aleatoria, definida por sorteo. Para la prueba de la AMB, el atleta debería lanzar un medicine ball de $3 \mathrm{Kg}$. a la máxima distancia posible. Para el análisis isocinético se utilizaron velocidades de 60\% y $180 \%$ para evaluar la capacidad de los músculos rotadores internos del hombro. Para analizar las asociaciones se utilizaron las pruebas de correlación de Pearson y regresión lineal. Resultados: Asociaciones moderadas $(0,60 \leq r<0,70 ; p<0,05)$ fueron observadas para las variables PT, PMy TTy para la prueba AMB a 60\% s. Además, las asociaciones entre las variables a 180\% fueron altas $(r \geq 0,70 ; p<0,05)$. Conclusión: Las variables isocinéticas PT, PM Y TT de los rotadores internos del hombro del brazo dominante de atletas de balonmano del sexo masculino mostraron una correlación moderada y alta con la prueba funcional AMB.

Palabras clave: hombro, torque, dinamómetro de fuerza muscular. 


\section{INTRODUÇÃO}

O handebol é uma modalidade esportiva caracterizada por esforços físicos de alta intensidade e de curta duração, destacando as capacidades motoras de velocidade e de força, principalmente, a força explosiva e a força rápida'. Além disso, utiliza-se de fundamentos técnicos e movimentações táticas específicas para a construção de jogadas que resultem em uma conclusão em direção à meta adversária. Para cumprir o objetivo da modalidade, que é a marcação de gols², o atleta de handebol usa um fundamento denominado arremesso ${ }^{3}$. Este complexo gesto esportivo, que depende do equilíbrio dinâmico entre os músculos rotadores internos e externos de ombro ${ }^{4}$, tem sido estudado a partir de variáveis biomecânicas tais como análise cinemática tridimensional5,6 e desempenho muscular ${ }^{7,8}$

Têm sido descrito na literatura testes indiretos para avaliar e quantificar o arremesso no handebol, segundo suas características de força, potência ${ }^{9}$ e precisão ${ }^{10}$. A dinamometria isocinética poderia ser a ferramenta para este tipo de avaliação, já que tem sido comumente utilizada na avaliação do comportamento muscular no âmbito científico ${ }^{11}$. Dentre as inúmeras possibilidades, essa ferramenta fornece informações sobre a capacidade muscular, avaliando-se principalmente torque, trabalho e potência ${ }^{12}$. O entrave está justamente no acesso a essa tecnologia, restringindo os treinadores ao acesso a dados importantes que poderiam melhorar efetivamente o desempenho de seus atletas dentro de quadra.

Nos últimos anos, diversos testes funcionais têm sido descritos para verificar o desempenho físico de atletas nas mais variadas modalidades esportivas ${ }^{13}$. Alguns estudos ${ }^{14,15}$ apresentam o teste de arremesso de medicine ball (AMB) como ferramenta prática e fácil na mensuração do nível de força para membros superiores. Especificamente em relação ao handebol, Dechechi et al. ${ }^{16}$ verificaram os efeitos de temporada de treinamento físico sistematizado de 38 semanas aplicado em uma equipe de handebol feminino sub-21 sobre o desempenho físico observado com testes funcionais, dentre eles, o AMB. Oliveira et al. ${ }^{17}$ utilizaram o AMB como uma das ferramentas para verificar o comportamento de variáveis neuromusculares, frente a um período de destreinamento em atletas de handebol.

Entretanto, ainda são poucas as informações que relacionem testes funcionais com testes mais precisos, como a dinamometria isocinética. Aliás, D'Alessandro et al. ${ }^{18}$ destacam a necessidade de estudos científicos para a fundamentação e a utilização destes testes, pois muitos não possuem validade comprovada. A validação de testes funcionais na avaliação da função muscular poderia fornecer informações úteis e de baixo custo, permitindo, por exemplo, selecionar atletas que necessitem de avaliação mais detalhada em equipamentos sofisticados, como o dinamômetro isocinético.

Desta forma, o presente estudo teve por objetivo correlacionar as variáveis isocinéticas pico de torque (PT), potência média (PM) e trabalho total (TT) de rotadores internos de ombro em atletas de handebol do gênero masculino com o teste funcional de AMB. Além disso, propor equações preditivas para as variáveis isocinéticas estudadas a partir do teste de AMB.

\section{MÉTODOS}

Participaram do estudo 25 atletas amadores de handebol do gênero masculino com idade média de 17,72 \pm 2,16 anos, altura média de $173,00 \pm$ 0,08 cm, massa corporal média de 70,13 $\pm 16,25$ kg e índice de massa corporal (IMC) de 23,23 $\pm 4,82 \mathrm{~kg} / \mathrm{m}^{2}$. Para serem incluídos no estudo os atletas não poderiam apresentar lesões nos ombros. Todos os atletas assinaram voluntariamente o termo de consentimento de participação nos testes. Além disso, todos os procedimentos experimentais adotados atendiam aos preceitos da Lei 196/96 do Conselho Nacional de Saúde, a qual estabelece as diretrizes e normas regulamentadoras de pesquisa envolvendo seres humanos (Parecer $n^{\circ}$ 369.330).
Os procedimentos experimentais aconteceram na quadra interna e no Laboratório Integrado de Tecnologias Aplicadas à Saúde e ao Esporte (LiTec) do Centro de Ciências Aplicadas à Educação e Saúde (CeCAES). O CeCAES é uma unidade do Instituto Federal de Educação, Ciências e Tecnologia do Sul de Minas Gerais (IFSULDEMINAS), Campus Muzambinho, MG, direcionada exclusivamente às atividades desenvolvidas pelo curso superior de Educação Física do IFSULDEMINAS, Campus Muzambinho.

A coleta de dados foi realizada em dois dias diferentes, respeitando intervalo mínimo de 48 horas entre as sessões. Antes de cada sessão experimental o avaliado realizou um aquecimento que consistia em cinco minutos de corrida e cinco minutos de movimentos específicos do handebol, tais como passes e arremessos. De maneira aleatória, definida por sorteio, os atletas faziam o teste isocinético ou o teste de AMB.

Para a medida da distância arremessada no teste de AMB foi utilizado o protocolo adaptado de Johnson e Nelson ${ }^{19}$. Foi solicitado ao atleta que realizasse o movimento de forma similar a um arremesso do handebol, usando apenas uma das mãos ${ }^{15}$. 0 atleta ficou sentado numa cadeira que estava encostada em uma parede, com os pés apoiados no solo (figura 1). Para diminuir a ação do impulso do tronco durante o arremesso e evitar compensações, o tronco do atleta ficou apoiado no encosto da cadeira seguro por uma faixa, na altura do peito. A bola de medicine ball de $3 \mathrm{~kg}$ foi segurada pelo atleta próxima à parede, com o cotovelo flexionado e abduzido a $90^{\circ}$, de onde se iniciava o movimento. Para garantir a angulação desejada, foi utilizado um flexímetro da marca Sanny. O atleta deveria lançar a bola a maior distância possível sobre a trena demarcatória. O resultado do teste foi a distância arremessada (em centímetros), entre a linha demarcatória desenhada entre os pés dianteiros da cadeira e o primeiro contato da bola de medicine ball sobre ou próximo à trena afixada no solo. Cada atleta fez três tentativas com a mão dominante, com intervalo mínimo de 3 minutos entre as tentativas. A média das três tentativas foi considerada o resultado do teste.

A avaliação isocinética foi realizada por um dinamômetro isocinético Biodex System 4 Pro (Biodex Medical Systems Inc., Nova lorque, EUA). Após prévio aquecimento, que consistiu em 5 minutos de corrida leve e 5 minutos de passes e arremessos com ambos os braços, os atletas realizavam uma série de três repetições nas velocidades de $60 \% \mathrm{~s}$ e $180^{\circ}$ s para familiarização com os testes. Os testes foram realizados na modalidade concêntrico/concêntrico e nas velocidades supracitadas, para rotadores internos e externos de ombro. O atleta foi posicionado assentado na cadeira do dinamômetro (figura 2). A angulação do encosto da cadeira foi de $85^{\circ}$ e o eixo da articulação do ombro foi

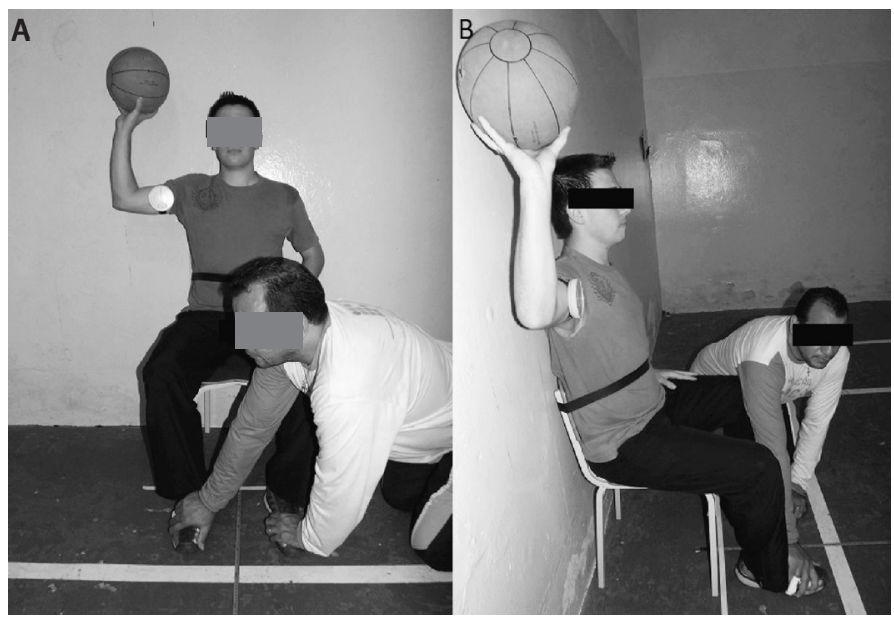

Figura 1. Vista frontal (A) e lateral (B) do teste de arremesso de medicine ball com uma das mãos adaptado de Johnson e Nelson ${ }^{19}$. 


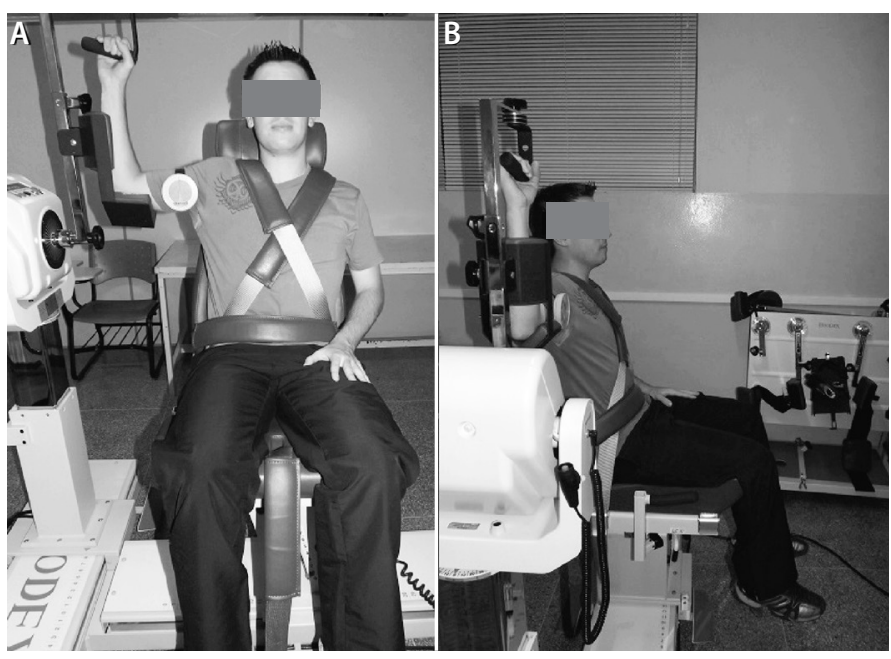

Figura 2. Vista frontal (A) e lateral (B) do teste isocinético para rotador interno de ombro.

alinhado com o eixo do dinamômetro. O ombro ficou abduzido a $90^{\circ}$. A amplitude de teste foi limitada em $75^{\circ}$, a partir da posição considerada inicial. Essa posição foi obtida da seguinte maneira: deslocamento de $90^{\circ}$ de abdução de ombro, flexão de $90^{\circ}$ de cotovelo e rotação externa de ombro de $90^{\circ}$. Com essa configuração, o membro dominante foi posicionado na alavanca isocinética conforme recomendação do fabricante. Entre cada velocidade era dado um intervalo de 30 segundos de repouso. Todos os testes foram realizados com o mesmo estímulo verbal, a fim de motivar o esforço máximo dos atletas durante a realização dos testes.

As variáveis isocinéticas avaliadas foram o pico de torque (PT), o trabalho máximo (TM) e o trabalho total (TT). Em relação às variáveis analisadas, cabe ressaltar que o PT e o TM são extraídos da melhor curva de torque que o sujeito produziu, enquanto que o TT corresponde à soma do trabalho muscular exercido nas três repetições executadas. Tais valores foram obtidos diretamente do arquivo fornecido pelo dinamômetro ${ }^{20}$.

\section{Análise estatística}

O teste de correlação de Pearson foi utilizado para a análise da associação entre as variáveis isocinéticas PT, TT e PM nas velocidades $60 \%$ e $180 \%$ s com a distância máxima arremessada. Foi adotado um nível de significância de $p<0,05$. O valor de $r$ foi considerado pequeno (até 0,25$)$, baixo $(0,26-0,49)$, moderado $(0,50-0,69)$, alto $(0,70$ a 0,89$)$ e muito alto (acima de 0,90 ), de acordo com os valores de referência descritos por Jhonson e Gross ${ }^{21}$.

Além disso, foram criadas as equações de predição a partir de análise de regressão linear simples ajustada pela distância do arremesso e as variáveis isocinéticas. O programa estatístico GraphPadPrism 5.0 foi utilizado para as análises bem como para a confecção dos gráficos.

\section{RESULTADOS}

A figura 3 apresenta as associações entre as variáveis isocinéticas e a máxima distância arremessada nas duas velocidades estudadas $(60 \%$ e 180\% s). Foram observadas associações moderadas $(0,60 \leq r<0,70$; $\mathrm{p}<0,05)$ para as variáveis PT, PM e TT e o teste de AMB na velocidade de $60^{\circ}$ s. Além disso, observaram-se associações altas ( $\left.r \geq 0,70 ; p<0,05\right)$ entre as variáveis estudadas na velocidade de $180^{\circ}$ s.

A tabela 1 ilustra as equações de predição do PT, PM e TT a partir da distância do arremesso no teste de AMB, bem como o erro de estimativa dessas equações. A variável y indica a variável isocinética predita e a variável $x$ equivale à distância arremessada no teste funcional.
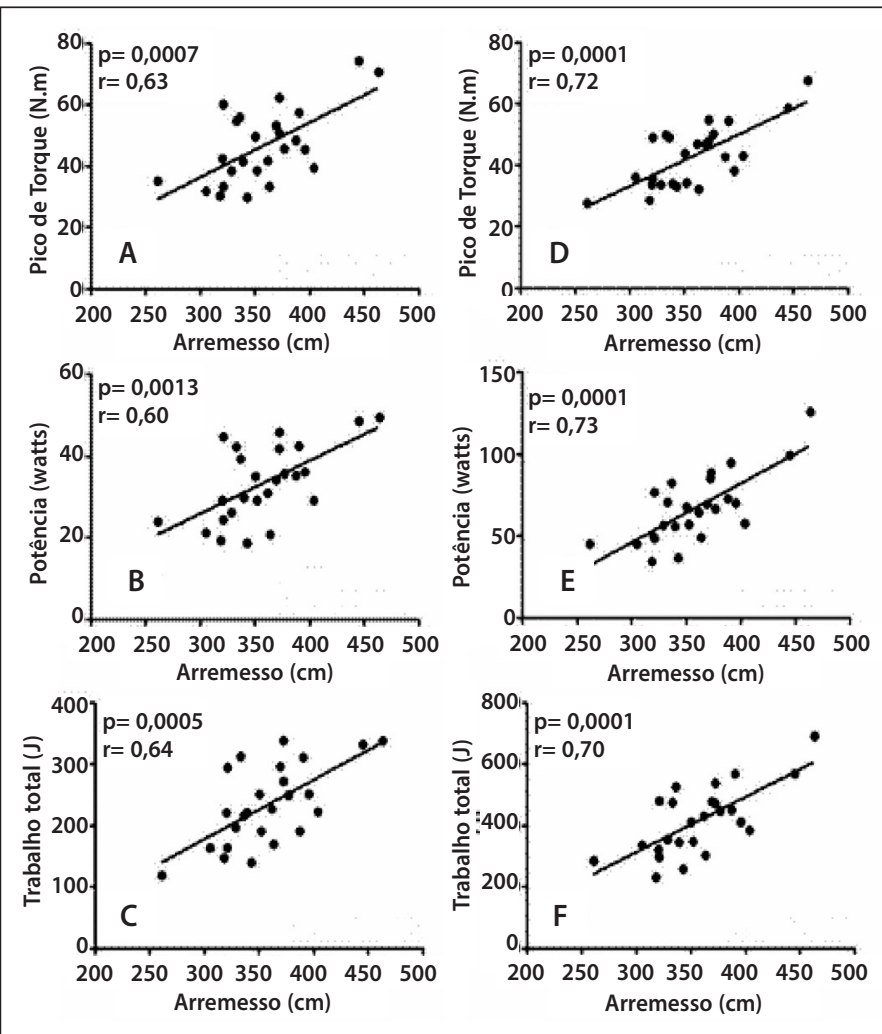

Figura 3. Associações entre as variáveis isocinéticas pico de torque, trabalho total e potência nas duas velocidades avaliadas (60\% /s: A, B e C; e 180\% / D, E e F) com a distância dos arremessos no teste funcional com medicine ball. $(n=25)$. 0 teste de correlação de Pearson revelou associações moderadas a altas com p<0,05 em todas as análises.

Tabela 1. Equações de predição de variável isocinética a partir de teste de arremesso com medicine ball.

\begin{tabular}{c|c|c}
\hline Variáveis de previsão & Equação de previsão & Erro da estimativa \\
\hline $\mathbf{6 0} / \mathbf{s}$ & & \\
\hline Pico de Torque (N.m) & $\mathrm{y}=0,178 x-17,27$ & $-0,25 \pm 9,52$ \\
\hline Potência Média (W) & $\mathrm{y}=0,129 x-12,94$ & $-0,16 \pm 7,35$ \\
\hline Trabalho Total $(\mathrm{J})$ & $\mathrm{y}=0,965 \mathrm{x}-111,6$ & $-0,13 \pm 50,23$ \\
\hline $\mathbf{1 8 0} / \mathbf{s}$ & & \\
\hline Pico de Torque (N.m) & $\mathrm{y}=0,168 \mathrm{x}-17,43$ & $-0,27 \pm 6,97$ \\
\hline Potência Média (W) & $\mathrm{y}=0,360 \mathrm{x}-62,21$ & $-0,25 \pm 14,30$ \\
\hline Trabalho Total $(\mathrm{J})$ & $\mathrm{y}=1,799 x-226,2$ & $0,02 \pm 79,58$ \\
\hline
\end{tabular}

\section{DISCUSSÃO}

A dinamometria isocinética tem sido o equipamento de escolha para uma avaliação pormenorizada e detalhada da capacidade muscular. Contudo, o alto custo desse equipamento restringe sua acessibilidade e intensifica a busca de novas ferramentas para avaliação do desempenho muscular. Sendo assim, diversos testes funcionais têm sido descritos e aplicados nos mais variados contextos esportivos ${ }^{13}$. Ao usar um teste funcional, os profissionais que lidam com esse dilema são favorecidos pelo baixo custo e relativa facilidade na obtenção de parâmetros importantes para a modulação do treinamento esportivo. Entretanto, é fundamental que os testes funcionais sejam validados e correlacionados com ferramentas mais precisas. Inserido nesse contexto, o propósito deste estudo foi correlacionar variáveis isocinéticas de rotadores internos de ombro de atletas de handebol com o teste de $A M B$, propondo equações preditivas a partir do teste de AMB.

No presente estudo observou-se moderada correlação durante a velocidade de 60\%/s (PT: $r=0,63 ;$ PM: $r=0,60$ e TT: $r=0,64$ ), e alta correlação durante a velocidade de 180\%/s (PT: $r=0,72$; PM: $r=0,73$ e TT: $r=0,70$ ) entre as variáveis isocinéticas e o teste AMB. A partir das equações de predição, foi identificado que o erro das estimativas variou de -013 a -0,25 
nos testes isocinéticos a 60\% s e -0,27 a 0,02 durante a velocidade de $180 \%$ s. Esses resultados representam um erro aproximado menor que $1 \%$. Dessa maneira, sugere-se que o teste de AMB possa ser usado para inferir sobre a capacidade muscular de rotadores internos. Segundo nosso conhecimento, é a primeira vez que esse teste é correlacionado com a dinamometria isocinética em atletas de handebol.

Fernandes et al. ${ }^{22}$ recomendam analisar os valores do coeficiente de determinação $\left(R^{2}\right)$ quando são geradas equações de predição. Em nosso estudo, foram encontrados valores de $R^{2}$ que oscilaram entre 0,40, 041 e 0,36 (60\%/seg) e 0,52, 0,49 e 0,54 (180\%/seg) para as equações de predição do PT, TT e PM, respectivamente. Assim, foi mostrado que a variável distância arremessada no teste de AMB determinou de 36\% a 54\% da variabilidade das variáveis isocinéticas estudadas. Dessa maneira, considerou-se que existem outros fatores causais que interferem com a capacidade muscular relacionada aos rotadores internos do ombro. Nesse sentido, é fundamental que as equações apresentadas no presente estudo sejam usadas com cautela principalmente em populações com características físicas distintas da amostra estudada. Outro fator que limita as generalizações dos resultados é a dificuldade técnica em garantir, durante o teste funcional, padrões biomecânicos de arremesso semelhantes entre os voluntários.

A avaliação isocinética de ombro em atletas de handebol tem sido documentada. Basicamente, os estudos caracterizam os picos de torque dos músculos rotadores externos e internos criando razões isocinéticas que poderiam identificar desequilíbrios, risco de lesão do manguito rotador e propondo estratégias de treinamento desses músculos ${ }^{4,23,24}$. Por outro lado, um grupo de pesquisadores gregos demonstrou que a variável isocinética pico de torque dos músculos rotadores de ombro não é um bom indicador da velocidade de qualquer tipo de arremesso, independente da habilidade técnica do jogador de handebol, exceto o arremesso com salto vertical ${ }^{8}$. Além disso, ficou evidenciado que a eficácia do arremesso do jogador de handebol em realizar o gol também não se correlaciona com o pico de torque isocinético do manguito rotador ${ }^{7}$. Dessa maneira, é importante considerar que ao prever a capacidade muscular isocinética a partir do teste de $A M B$, não se espera a obtenção de parâmetros para a velocidade da bola durante o gesto de arremessar, tampouco da eficácia do arremesso.

Usando uma proposta metodológica semelhante apresentada no presente estudo, ou seja, correlacionar um determinado teste funcional

\section{REFERÊNCIAS}

1. Souza J, Gomes AC, Leme L, Silva SG. Alterações em variáveis motoras e metabólicas induzidas pelo treinamento durante um macrociclo em jogadores de handebol. Rev Bras Med Esporte.2006;12(3):129-34.

2. Reis HHB. O ensino do handebol utilizando-se do método parcial. EF Deportes. 2006;10(93).

3. Greco PJ, Romero JJF. Manual de handebol: da iniciação ao alto nível. São Paulo: Phorte, 2012

4. Edouard P, Degache F, Oullion R, Plessis JY, Gleizes-Cervera S, Calmels P. Shoulder strength imbalances as injury risk in handball. Int J Sports Med. 2013;34(7):654-60

5. Montes FA, Dezana DB, Santosa DC, Martinia E, Zimmerman CA, Gomes SC. Análise Tridimensional do Arremesso com Apoio no Handebol. UNOPAR Cient Ciênc Biol Saúde. 2012;14(1):5-8.

6. Tilaar R, Ettema G. A three-dimensional analysis of overarm throwing in experienced handball players. J Appl Biomech. 2007;23(1):12-9

7. Zapartidis I, Gouvali M, Bayios I, Boudolos K. Throwing effectiveness and rotational strength of the shoulder in team handball. J Sports Med Phys Fitness. 2007;47(2):169-78.

8. Bayios IA, Anastasopoulou EM, Sioudris DS, Boudolos KD. Relationship between isokinetic strength of the internal and external shoulder rotators and ball velocity in team handball. J Sports Med Phys Fitness. 2001;41(2):229-35.

9. Eleno T, Barela JA, Kokubun E. Tipos de esforço e qualidades físicas do handebol. Rev Bras Cienc Esporte. 2002;24(1):83-98

10. Nogueira MQ, Del Vecchio FB. Efeitos do treino de flexibilidade e força funcional na precisão em teste de handebol. Conexões. 2008;6(0):122-31.

11. Gleeson NP, Mercer TH. The utility of isokinetic dynamometry in the assessment of human muscle function. Sports Med. 1996;21:18-34.

12. Abernethy P, Wilson G, Logan P. Strength and power assessment: Issues, controversies and challenges. Sports Med. 1995;19:401-17.

13. Silva FF, Souza RA, Couto CLB, Magalhães RF, Kawatake JP. Efeitos agudos do alongamento estático no rendimento de testes funcionais em atletas de voleibol e futebol. Ter Man. 2011;9(42):138-42

14. Candotti CT, Soares VR, Noll M. A Influência da postura sobre as capacidades motoras: agilidade, força e velocidade. Rev Bras Ci e Mov. 2010;18(2):11-8 com resultados isocinéticos, Selistre et al. ${ }^{25}$ procuraram identificar a relação entre o torque extensor e a relação isquiotibiais/quadríceps com o salto unipodal triplo horizontal em jogadores profissionais de futebol. Por sua vez, D'Alessandro et al. ${ }^{18}$ verificaram se existia a associação entre o hop test e dados da função muscular fornecidos pela avaliação do joelho no dinamômetro isocinético em atletas profissionais. Em ambos os estudos, os autores encontraram uma baixa correlação entre os testes funcionais com a dinamometria isocinética, indicando que o salto unipodal triplo horizontal e o hop test não devem ser usados para substituir o dinamômetro na avaliação da função muscular. Ao contrário, os resultados aqui apresentados mostraram que o teste de AMB possui uma correlação moderada com as variáveis isocinéticas dos rotadores internos de ombro, indicando que esse teste pode ser uma maneira prática e útil para identificação e previsão da capacidade muscular de jogadores de handebol.

\section{CONCLUSÃO}

Deve-se ressaltar que esse estudo não permite predizer o risco de lesão do manguito rotador ou elaborar estratégias de treinamento somente com os dados do teste de AMB. Além disso, novos estudos são necessários para melhor esclarecer essa questão. É fundamental a identificação de outras variáveis causais para a elaboração de equações mais complexas e que usem análises de regressão múltipla.

Por fim, as variáveis isocinéticas PT, PM e TT, de rotadores internos de ombro do braço dominante de atletas de handebol do gênero masculino apresentam correlação moderada a alta com o teste funcional de AMB.

\section{AGRADECIMENTO}

O autor Fabiano Fernandes da Silva agradece ao Instituto Federal de Educação, Ciência e Tecnologia do Sul de Minas Gerais - IFSULDEMINAS, ao apoio financeiro recebido para a publicação deste artigo científico e à Coordenação de Aperfeiçoamento de Pessoal de Nível Superior (CAPES) pela concessão da bolsa de doutorado no Programa de Pós-Graduação em Engenharia Biomédica da Unicastelo, São José dos Campos/SP, Brasil.

Todos os autores declararam não haver qualquer potencial conflito de interesses referente a este artigo.
15. Ikeda Y, Kijima K, Kawabata K, Fuchimoto T, Ito A. Relationship between side medicine-ball throw performance and physical ability for male and female athletes. Eur J Appl Physiol . 2007;99:47-55.

16. Dechechi CJ, Machado EFA, Ide BN, Lopes CR, Brenzikofer R, Macedo DV. Estudo dos Efeitos de Temporada de Treinamento Físico Sobre a Performance de Uma Equipe de Handebol Feminino Sub-21. Rev Bras Med Esporte. 2010;16(4):295-300.

17. Oliveira VL, Leite GS, Leite RD, Assumpção CO, Pereira GB, Neto JB, Prestes J. Efeito de um período de destreinamento sobre variáveis neuromusculares em atletas de handebol. Fit Perf J. 2009;8(2):96-102

18. D'Alessandro RL, Silveira EAP, Anjos MTS, Silva AA, Fonseca ST. Análise da associação entre a dinamometria isocinética da articulação do joelho e o salto horizontal unipodal, hop test, em atletas de voleibol. Rev Bras Med Esporte. 2005;11(5):271-5.

19. Johnson BL, Nelson JK. Practical measurements for evaluation in physical education. Minnesota: Burgess, 1979

20. Anselmo GN, Elisangela FM. Influência do Volume de Alongamento Estático dos Músculos Isquiotibiais nas Variavéislsocinéticas. Rev Bras Med Esporte. 2009;15(2):104-9.

21. Jonson SR, Gross MT. Intraexaminer Reliability, Interexaminer Reliability, and Mean Values for Nine Lower Extremity Skeletal Measures in Healthy Naval Midshipmen. J Orthop Sports Phys Ther .1997; 25(4):253-63.

22. Fernandes $A A$, Silva CD, Vieira BC, Marins JCB. Validade preditiva de equações de referência para força de preensão manual em homens brasileiros de meia idade e idosos. Fisioter Pesq. 2012;19(4):351-6.

23. Andrade MS, Fleury AM, de Lira CA, Dubas JP, da Silva AC. Profile of isokinetic eccentric-to-concentric strength ratios of shoulder rotator muscles in elite female team handball players. J Sports Sci 2010;28(7):743-9.

24. Andrade MS, Lira CA, Vancini RL, de Almeida AA, Benedito-Silva AA, da Silva AC. Profiling the isokinetic shoulder rotator muscle strength in 13- to 36-year-old male and female handball players. Phys Ther Sport. 2013;14(4):246-52

25. Selistre LFA, Cintra GC, Aleixo Júnior RD, Rosa SMMG. Relação entre torque extensor e relação I:Q com salto unipodal triplo horizontal em jogadores de futebol. Rev Bras Med Esporte. 2012;18(6):390-3. 